\title{
Neutralized solar energetic particles for SEP forecasting: Feasibility study of an innovative technique for space weather applications
}

\author{
Xiao-Dong Wang ${ }^{1 *}$, B. Klecker ${ }^{2}$, G. Nicolaou ${ }^{1}$, S. Barabash ${ }^{1}$, M. Wieser ${ }^{1}$, P. Wurz ${ }^{3}$, A. Galli ${ }^{3}$, \\ F. Cipriani ${ }^{4}$, and Y. Futaana ${ }^{1}$ \\ 'Swedish Institute of Space Physics, Kiruna, Sweden; \\ ${ }^{2}$ Max-Planck Institute für extraterrestrische Physik, Garching, Germany; \\ ${ }^{3}$ Physics Institute, University of Bern, Bern, Switzerland; \\ ${ }^{4}$ ESA/ESTEC, Research and Scientific Support Department, Noordwijk, The Netherlands
}

Key Points:

- Neutralized solar energetic particles may hit the Earth hours earlier than the SEPs, suggesting the potential for space weather forecasting.

- Their temporal and spectral properties at Earth depend on the characteristics of a CME-driven shock.

- Corresponding measurement requirements are formulated, which are achievable with an instrument in Earth orbit using mature technology.

Citation: Wang, X.-D., Klecker, B., Nicolaou, G., Barabash, S., Wieser, M., Wurz, P., Galli, A., Cipriani, F., and Futaana, Y. (2022). Neutralized solar energetic particles for SEP forecasting: Feasibility study of an innovative technique for space weather applications. Earth Planet. Phys., 6(1), 42-51. http://doi.org/10.26464/epp2022003

\begin{abstract}
Energetic neutral atoms (ENAs) are produced by the neutralization of energetic ions formed by shock-accelerated gradual solar energetic particle events (SEP). These high-energy ENAs (HENAs) can reach the Earth earlier than the associated SEPs and thus can provide information about the SEPs at the lower corona. The HENA properties observed at Earth depend on the properties of the coronal mass ejection (CME)-driven shocks that accelerate the SEPs. Using a model of HENA production in a shock-accelerated SEP event, we semi-quantitatively investigate the energy-time spectrum of HENAs depending on the width, propagation speed, and direction of the shock, as well as the density and ion abundances of the lower corona. Compared to the baseline model parameters, the cases with a wider shock width angle or a higher coronal density would increase the HENA flux observed at the Earth, while the case with an Earthpropagating shock shows a softened HENA spectrum. The comparison of expected HENA fluxes in different cases with a flight-proven ENA instrument suggests that solar HENAs can feasibly be monitored with current technologies, which could provide a lead time of 2-3 hours for SEPs at a few MeV. We propose that monitoring of solar HENAs could provide a new method to forecast shock-driven SEP events that are capable of significant space weather impacts on the near-Earth environment.
\end{abstract}

Keywords: solar energetic particles; energetic neutral atoms; space weather; numerical simulation

\section{Introduction}

Energetic neutral atoms (ENAs) exist widely in the Solar System. ENAs are produced via charge exchange wherever ions coexist with charge-neutralizing materials (e.g., neutral gases, neutral surfaces, or dense plasma gas). Theoretically, ENAs can be produced in the immediate environment of the Sun. Therefore, ENA diagnostics can be considered as a potential monitoring tool for space weather events. Hsieh et al. (1992) first proposed monitoring ENAs produced by a coronal mass ejection (CME) to give advance warning of the arrival at Earth of an interplanetary CME (ICME). Gruntman (1994) further expanded the model of Hsieh et

Correspondence to: X.-D. Wang, wang@irf.se

Received 22 JUN 2021; Accepted 23 SEP 2021.

Accepted article online 06 DEC 2021.

(C) 2022 by Earth and Planetary Physics. al. (1992) and compared the expected flux with that of the neutral solar wind. However, these predictions did not cover ENA-generating processes close to the Sun $\left(<15 R_{\mathrm{S}}\right)$, and they did not consider energy ranges higher than $20 \mathrm{keV} /$ nuc.

Mewaldt et al. (2009) reported observations, by the Low Energy Telescopes (LETs) on both Solar TErrestrial RElations Observatory (STEREO) A and B spacecraft, of signals corresponding to $1 \mathrm{MeV}$ particles that came from the Sun's direction during a solar flare event on 5 December 2006. The flux arrived within $\pm 10^{\circ}$ of the direction from the solar equator, and matched very well with the profile of the X-ray intensity from the same flare, leading to the conclusion that the recorded signals were due to $1 \mathrm{MeV}$ hydrogen ENAs that originated from recombination and charge exchange $(C X)$ in the solar corona. Such high-energy ENAs (HENA) may open a new window for monitoring and studying the Sun and our near-space environment. 
To verify the conclusions of Mewaldt et al. (2009), Wang LH et al. (2014) proposed a simplified model to predict the properties of solar HENAs. The model consists of a spherical-cap-shaped shock driven by a CME erupting at $t=0$. The shock forms at a heliocentric distance of $1.5 R_{\mathrm{S}}$ with a variable width angle, and propagates in the direction perpendicular to the Sun-observer line. The shock energizes a fraction of the coronal protons, generating isotropic SEP emission. Some SEP protons exchange their charges with coronal $\mathrm{C}^{4+}$ and $\mathrm{O}^{6+}$ ions, forming HENAs later observed in orbit around the Earth. The modelled characteristics of solar HENAs agree qualitatively with the observations reported by Mewaldt et al. (2009), indicating that such a simplified empirical model of a CME shock and its interaction with coronal plasma is capable of reproducing the key parameters of solar HENAs (measured under the specified conditions of observation), thus supporting the usefulness of such solar HENA measurements in forecasting SEP events.

To evaluate the applicability of this idea, the next step, and the goal of this study, is to investigate the dependencies of the solar HENA properties on the properties of the shock, and whether solar HENAs can be detected by a typical ENA instrument under certain conditions. This investigation is done by modelling the solar HENA properties under different upstream conditions and comparing them with the performance of a typical HENA instrument. For the modelling part, we expand, beyond the work of Wang $\mathrm{LH}$ et al. (2014), the ranges of several parameters describing the production and propagation of HENAs, and investigate the variability of solar HENA properties depending on these parameters. For the detection feasibility part, although the STEREO/LETs observed the possible solar HENA signals, they are not ENA instruments, and there has been no dedicated solar HENA instrument in space. We thus compare the solar HENA properties with data from Cassini/INCA, a flight-proven reference instrument with measurement capabilities compatible with the expected HENAs.

\section{Methodology}

We reproduce the HENA production and propagation model from Wang LH et al. (2014) as the baseline model for this study, changing some parameters to investigate variations of the expected HENA properties that can be observed. No cross-variation relations (two or more parameters deviating from the baseline values at the same time) are evaluated. Nevertheless, the changed parameters have in many cases effects that are independent of each other, and therefore the effects of combined parameter changes are straightforward and understandable.

\subsection{The Baseline Model}

The baseline model (Wang LH et al., 2014) consists of a partially spherical-shaped shock front formed at a heliocentric distance of $1.5 R_{\mathrm{S}}$ with a width angle of $60^{\circ}$ at $t=0$, which then propagates in the direction perpendicular to the Sun-observer line with a speed of $1800 \mathrm{~km} / \mathrm{s}$ (Figure 1). The coordinate system is centered on the Sun, with the $x$-axis pointing to the observer at the Earth, the $x-y$ plane containing the symmetry axis of the cone that defines the CME. Therefore the direction of the central line of shock propagation is orthogonal to the Earthward direction. Hereafter, we refer to the volume inside the cone centered at the Sun and between

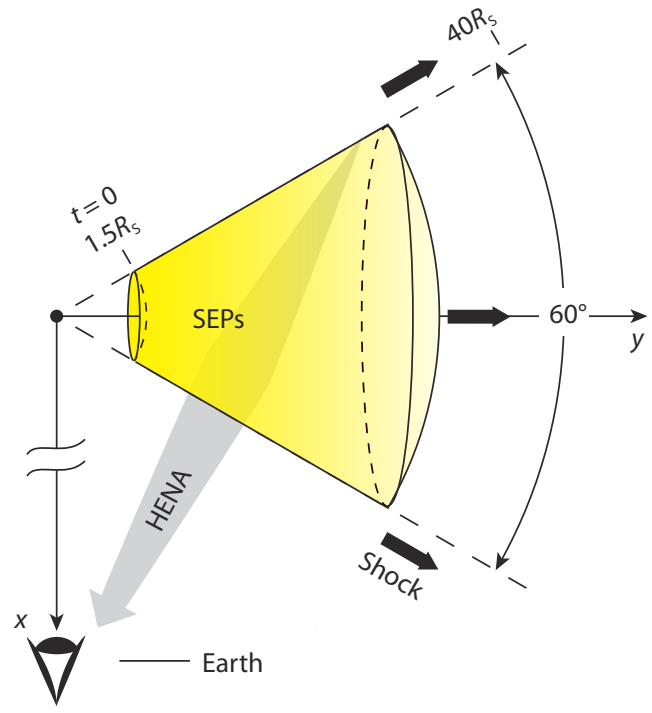

Figure 1. Illustration of the standard HENA production model used in this work. Adopted from Wang LH et al. (2014). HENA source region shown in yellow.

the heliocentric distances of $1.5 R_{\mathrm{S}}$ and $40 R_{\mathrm{S}}$ as "the HENA source region" (shaded yellow in Figure 1).

As the shock sweeps across the source region, it energizes protons in the background solar wind. The background solar wind flows at a speed of $450 \mathrm{~km} / \mathrm{s}$. The omnidirectional distribution function of shock-accelerated protons in the frame downstream of the shock is (Lee, 2005):

$$
f_{p}(v)=\frac{\beta a n_{\mathrm{SW}}}{4 \pi v_{0}^{3}}\left(\frac{v}{v_{0}}\right)^{-\beta}
$$

where $v$ is the speed in the downstream frame, $\beta=3 s /(s-1)$ is the power-law index of the SEP protons, $s=3.5$ is the compression ratio of the shock, $a=0.01$ is the fraction of CME protons injected into the diffusive shock acceleration process, and $v_{0}=2000 \mathrm{~km} / \mathrm{s}$ is the injection speed. We consider the energized protons from 5 keV to $20 \mathrm{MeV}$ in this study. The solar wind density $n_{\text {sw }}$ decays with the increasing heliocentric distance as:

$$
n_{\mathrm{SW}}= \begin{cases}10^{7.40-8.80 x+7.68 x^{2}-3.12 x^{3}}, & \text { if } x>1.0, \\ 1.46 \times 10^{5-2 x}, & \text { otherwise },\end{cases}
$$

where $x=\log _{10} r$, and $r$ is the heliocentric distance in solar radii $R_{S}$ (Allen et al., 2000). As the shock propagates, the distribution function of the shocked protons in the downstream region is averaged across the downstream region, maintaining the conservation of the total number of protons.

We use the distribution of shocked SEPs to calculate the production rate of HENAs in the downstream region of the shock. We consider the protons' $\mathrm{CX}$ with $\mathrm{O}^{6+}, \mathrm{C}^{4+}$, and the neutral hydrogen in the solar wind as the production processes. The relative abundances of the three species are respectively: $\mathrm{O}^{6+} / \mathrm{p} 10^{-3}, \mathrm{C}^{4+} / \mathrm{O}^{6+}$ $\sim 0.067$ (von Steiger et al., 2000), H/p $2.6 \times 10^{-7}$ (D'Amicis et al., 2007). We then consider three HENA loss processes: 1) photoionization, 2) proton and electron impact ionization, and 3) CX with solar wind protons along their trajectories. 


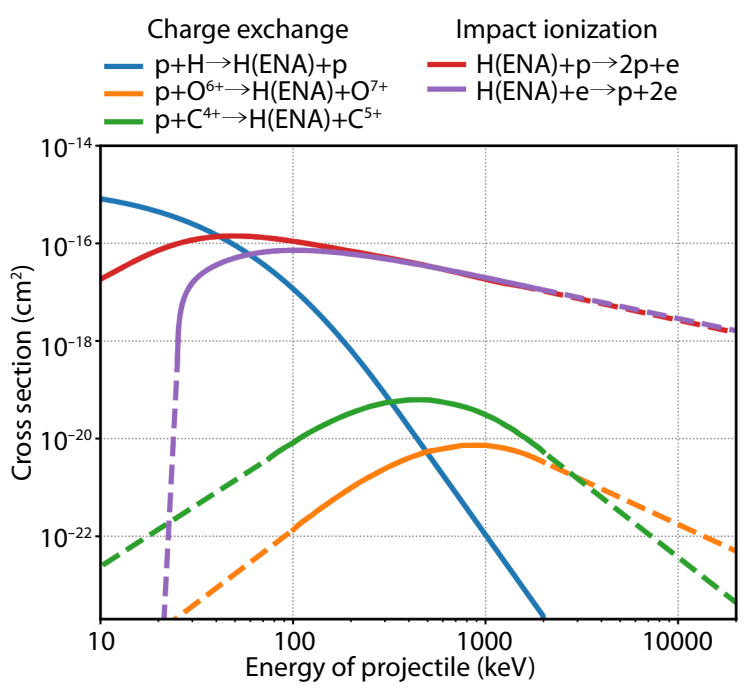

Figure 2. Cross sections used in this work. Dashed parts on curves show the power-law-extrapolated values outside the energy range of original references.

Figure 2 shows the cross sections of ENA production and loss processes used in this study. The documented energy ranges for $C X$ reactions with $\mathrm{O}^{6+}$ and $\mathrm{C}^{4+}$ are not sufficient for this study (Kuang $Y R, 1992)$. We therefore extrapolate the cross sections to the required energy range using a power law (dashed lines). The cross section of CX with neutral hydrogen in the solar wind is from Gruntman (1997). The electron recombination is neglected for the HENA production processes due to its small reaction rate ( $\sim 5$ orders of magnitude smaller) at this energy range (Andersen and Bolko, 1990). The proton impact ionization rate is from Barnett et al. (1990). These cross sections are used to compute ionization rates, assuming that the speed of the protons is much higher than the solar wind speed. This is correct for solar wind ions, while for solar wind electrons, it is only a zero-order approximation due to their much smaller mass.
The dependence of loss rate on the travel distance of ENAs is accounted for by the survival probability of ENAs (Gruntman et al., 2001) at location $l$ :

$$
h(l, v)=\mathrm{e}^{-\int_{0}^{l} \sum_{i} \gamma_{i}\left(l^{\prime}\right) \frac{\mathrm{d} l^{\prime}}{v}}
$$

where $\gamma_{i}$ is the loss rate of the individual loss process, and the integration path ranges from the birthplace of HENAs to the observer.

Figure 3 shows HENA fluxes seen at the Earth within a $12^{\circ} \times 180^{\circ}$ field of view. In the model, the HENA source region at any moment is the region behind the shock front, and is discretized into multiple lines of sight (LOS), converging at the Earth, along which the HENA flux is calculated. The HENA flux along a LOS is shown as a circular pixel in each panel of Figure 3. The four panels thus show the images of 100-keV HENAs observed between 9.5 to 14 hours after the onset of $\mathrm{CME}$, using standard parameters (see Table 1 for details). The front of the HENA source region appears as a different shape from the shock front (which is an arc centered at the Sun in our model). This is because of the different lengths of flying path, and thus travel times to Earth, between the HENAs produced at the closest and the farthest edges of the source region. At higher energies, the shock front and the front of the HENA source region become more similar in shape.

\subsection{Parameter Space in This Study}

Some parameters in the baseline model are highly variable in reality. To understand the dependence of solar HENA properties on these parameters, we altered the values of some model parameters to cover wider ranges of their respective distributions.

Table 1 lists all model parameters, their baseline model values, and additional values used in this study.

\section{The SEP model}

For the SEP production model, we keep the spherical-cap shape of

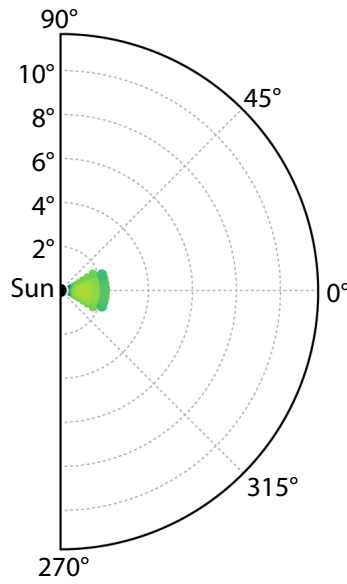

$10.08 \mathrm{~h}$

$8.05 \times 10^{-5} \mathrm{~cm}^{-2} \mathrm{~s}^{-1} \mathrm{keV}^{-1}$

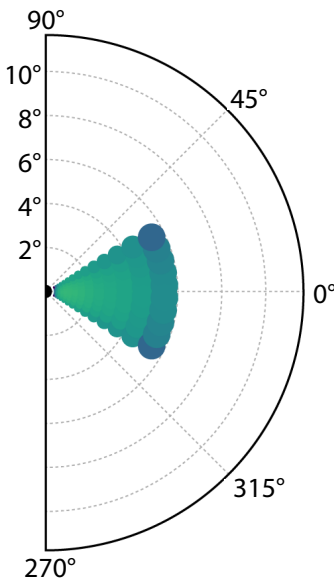

$11.39 \mathrm{~h}$

$3.24 \times 10^{-5} \mathrm{~cm}^{-2} \mathrm{~s}^{-1} \mathrm{keV}^{-1}$

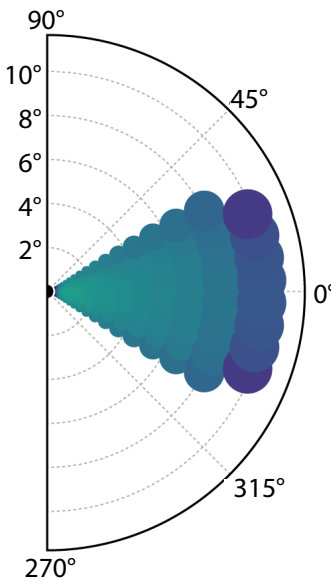

$12.88 \mathrm{~h}$

$1.84 \times 10^{-5} \mathrm{~cm}^{-2} \mathrm{~s}^{-1} \mathrm{keV}^{-1}$

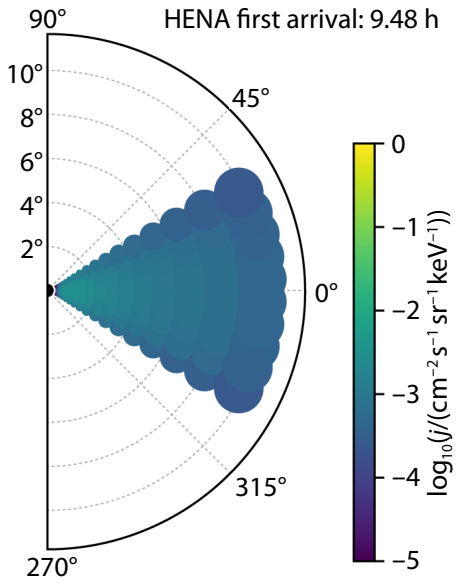

$13.97 \mathrm{~h}$

$1.27 \times 10^{-5} \mathrm{~cm}^{-2} \mathrm{~s}^{-1} \mathrm{keV}^{-1}$

Figure 3. 100-keV ENA differential directional flux images observed at the Earth at different times from the onset of the CME in the solar corona. The co-centered circular grid shows the angle (in degrees) from the solar center direction. The angles next to the arc border are the azimuth in the FOV. The black dot at the center of each polar plot is the solar disk to scale $\left(1 R_{\mathrm{s}}\right)$. The HENA flux value at the bottom of each panel is the integrated flux in that panel. 
Table 1. All model parameters and their values used in this study. The parameters with "n/a" represent the ones we kept at baseline model values.

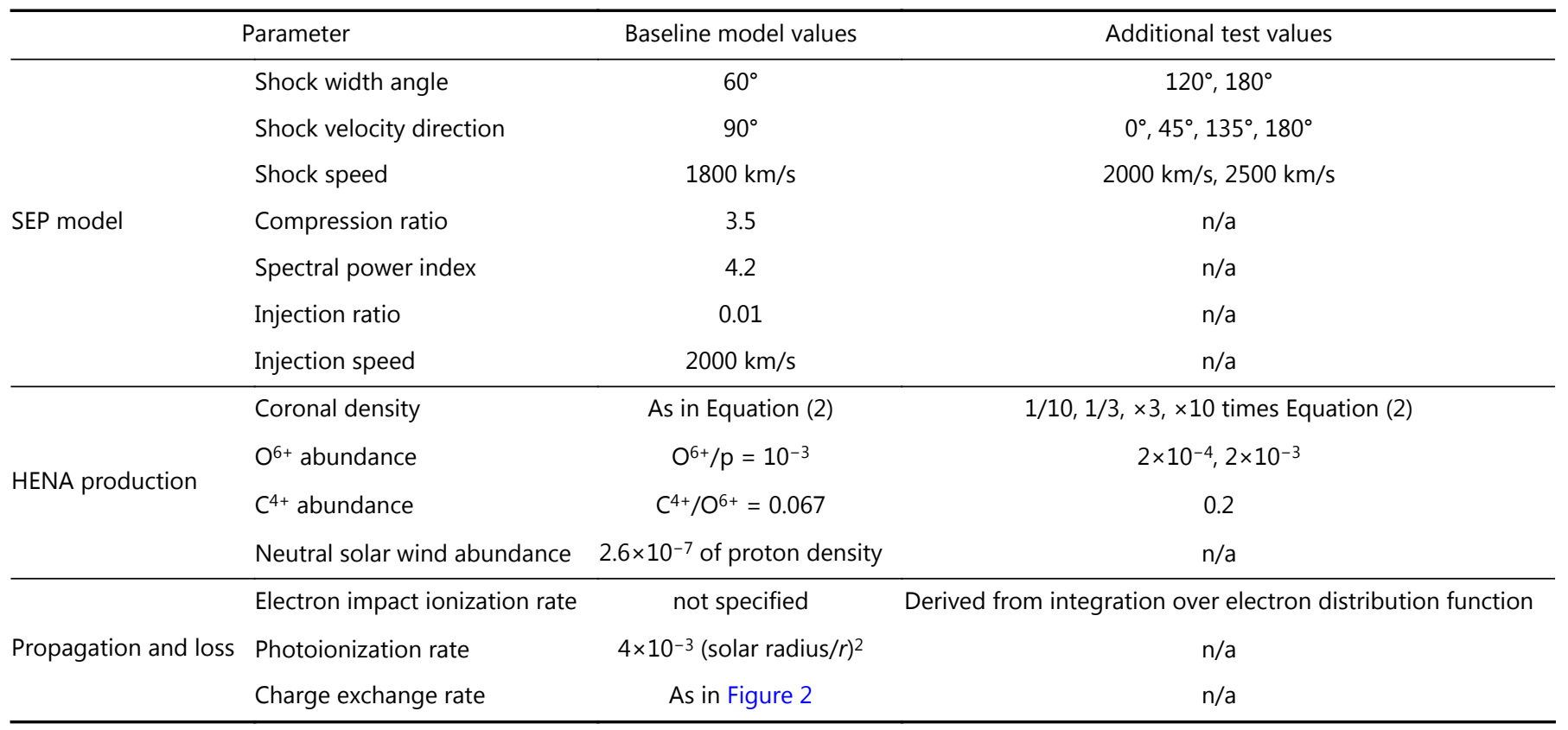

the shock and the $1.5-40 R_{\mathrm{S}}$ radius range of the source region but vary other geometric parameters:

(1) The width angle. The baseline model used $60^{\circ}$ as the width angle of the shock cone, which is relatively narrow for CMEs producing SEP events. A more realistic value would be $\sim 180^{\circ}$ (hemispherical), in particular for strong SEP events (Lamy et al., 2019).

(2) Shock velocity direction. In the baseline model, the shock propagates perpendicularly to the line of sight (shock velocity direction $90^{\circ}$ from the Sun-Earth line); in a general case, this direction is obviously unconstrained. We thus test the directions from the Earthward ( $0^{\circ}$ direction) to anti-Earthward ( $180^{\circ}$ direction) in $45^{\circ}$ intervals.

(3) The shock speed is determined by the propagation speed of the CME that drives the shock, which has a large event-to-event variation. Different shock speeds will change the time series of arriving HENAs at the observer. Since the shock speed is positively correlated with SEP peak intensity (Kahler, 2001), we test even higher shock speeds of 2000 and $2500 \mathrm{~km} / \mathrm{s}$.

\section{HENA production}

(1) The coronal density model. The coronal density can be \pm 1 order of magnitude different from the profile used in the baseline model, particularly in the low corona (Wurz and Gabriel, 1999). The local density determines the optical depth of HENA production and loss processes, and the resultant HENA flux is therefore not simply proportional to the column density along the line of sight. To address this spatiotemporal variability, we must use different density profiles and scaling factors to explore systematically the influence of the coronal density profile on ENA fluxes.

(2) Heavy ion abundances. The abundance of heavy ions relative to protons shows large temporal variations. The $\mathrm{O}^{6+}$ abundance ranges from $2 \times 10^{-4}$ to $2 \times 10^{-3}$ of the proton density (von Steiger et al., 2000). The $\mathrm{C}^{4+}$ abundance varies by a factor of $2-3$. The in- fluence of these abundance variations on Energetic Neutral Hydrogen (ENH) flux needs to be explored systematically.

\section{Propagation and loss of HENAs}

We follow the method used by Wang LH et al. (2014), considering the photoionization, and proton and electron impact ionization losses for propagating HENAs. Wang LH et al. (2014) did not specify how the electron velocity distribution was considered to apply to the impact ionization cross section. We thus compute the ionization rate by integration over a Maxwellian velocity distribution of solar wind electrons.

\section{Results}

\subsection{Properties of HENAs in the Baseline Model}

Figure 4 shows the results of the baseline simulation model in a format that will appear repeatedly in the following sections. Figure 4a presents the solar HENA omnidirectional flux at different energies observed at the Earth as a function of time after the CME initiation. The omnidirectional flux at the Earth is calculated by integrating the emitted differential directional flux over the whole production region seen from the Earth, as a function of time. As a comparison, the estimated arrival times of SEPs at the same energies as the HENAs are shown as vertical dashes at the top of Figure 4a. We adopted the $\sim 3$ hour travel time for $1.8 \mathrm{MeV}$ protons in the STEREO observation (Mewaldt et al., 2009), and scaled the travel time with SEP energies assuming that the SEP trajectories remain the same. Figure $4 \mathrm{~b}$ shows the energy spectra of the total fluence of the HENAs, distinguishing between the different production processes. The dominant production processes above $\sim 100 \mathrm{keV}$ are the $\mathrm{CX}$ with coronal $\mathrm{C}^{4+}$ and $\mathrm{O}^{6+}$ ions; below $100 \mathrm{keV}$, the $\mathrm{CX}$ with coronal and solar wind neutral $\mathrm{H}$ atoms dominates.

As a result, in the baseline model the travel times of HENAs from shock initiation to Earth arrival is always $\sim 80 \%$ of that of their par- 

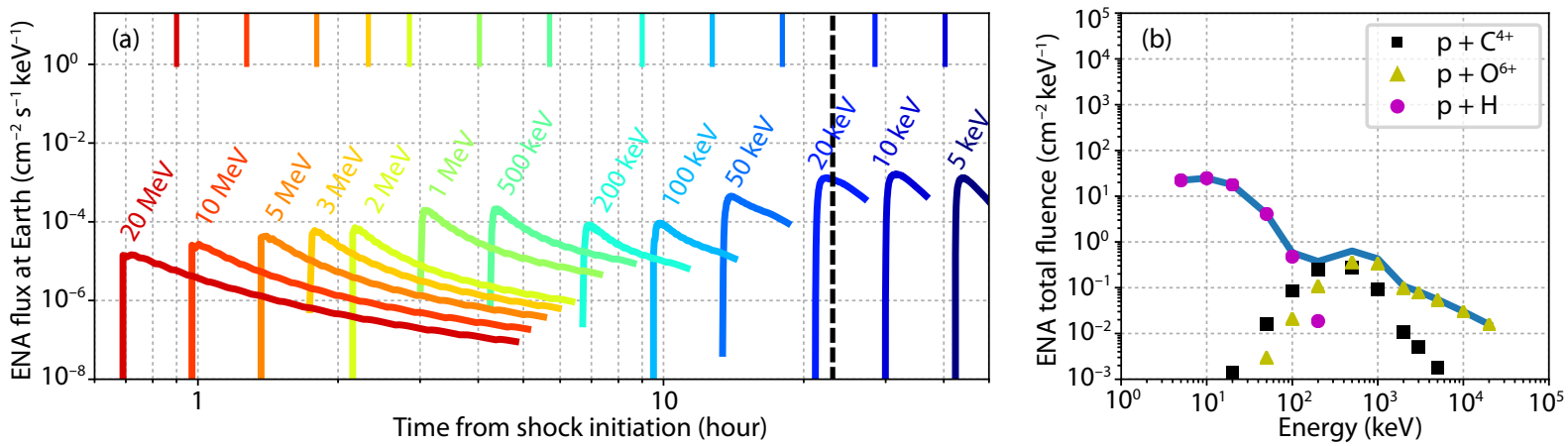

Figure 4. (a) Time series of HENA fluxes at different energies shown as curves of different colors. The time is plotted in log scale to emphasize the early times. The vertical dashes on the top show the arrival times of the SEPs with the energies coded in the same colors. The black vertical dashed line close to 22 hours shows the time of the shock reaching 1 AU distance from the sun. (b) The energy spectrum of the total fluence of observed HENAs. Three production processes are considered: charge exchange with $\mathrm{C}^{4+}, \mathrm{O}^{6+}$, and neutral $\mathrm{H}$ in the corona and the solar wind.

ent SEPs with the same energy. This travel time ratio is independent of particle energy, because it is due to the distance ratio of HENAs' straight-line path to the SEPs' path along the IMF line connecting the shock origin and the Earth. Since the IMF lines between the sun and the Earth are never completely radial, the solar HENAs will always arrive earlier than their parent SEPs of the same energy, the specific lead time between the HENA and SEP arrivals depending on the field line configuration. In the simulation results, the 20-MeV HENAs arrive 12 minutes earlier than the 20-MeV SEPs (0.7 hours vs. 0.9 hours after shock onset), suggesting a possible additional forecast proxy for SEP events, beyond such current proxies as the arrival of relativistic electrons.

In this paper, we refer for convenience to the HENA population with energies higher than $1 \mathrm{MeV}$ as "the SEP HENAs" since they are more relevant to the SEPs related to spacecraft operations than less energetic HENAs. Table 2 summarizes baseline model properties of SEP HENAs as observed at the Earth. The angular spread of the HENAs seen at the Earth is just the solid angle of the source region behind the shock, as shown in Figure 1, because HENAs move in straight lines. As the shock expands, the angular spread of the source region increases, hence so does the HENA angular spread. The HENA angular spread determines the angular resolution requirement of an ENA imager. The flux of HENAs is integrated over the full angular spread, and is time- and energy-dependent. We describe its temporal property by the time span between the arrival of the first flux peak at the highest simulated energy ( $20 \mathrm{MeV}$ ) and the arrival of the $20 \mathrm{keV}$ ENAs, whose speed is similar to that of the shock. We describe the HENA intensity by the range of the (temporal) peak fluxes of different energies. We will compare these properties as computed in the baseline model with results from other cases.

\subsection{Summary of All Results of Parameter Studies}

Table 3 summarizes the flux ranges of the SEP HENAs. The flux ranges from the baseline model results are colored as white, while those higher (lower) than the baseline model results are shown as different shades of green (red).

Among all runs, the ones with a wider width angle of the source region and those with a higher coronal density can produce higher differential fluxes at the Earth. Contrary to intuition, the case with a shock propagation angle $0^{\circ}$ (shock towards the Earth) does not show enhancement of differential fluxes. We will describe these cases in the following sections.

\subsection{Wider Shock Width Angle}

Figure 6 shows the model and results of the case with a $120^{\circ}$ shock width angle (Figure 5, Table 4). The wider width angle significantly increases the ENA production rate. The flux of SEP HENAs in this case ranges from $\sim 2 \times 10^{-4} \mathrm{~cm}^{-2} \mathrm{~s}^{-1} \mathrm{keV}^{-1}$ at $20 \mathrm{MeV}$ to $10^{-3}$ $\mathrm{cm}^{-2} \mathrm{~s}^{-1} \mathrm{keV}^{-1}$ at $1 \mathrm{MeV}$. These values are $\sim 5-10$ times greater and also reach the Earth a few minutes earlier than those in the

Table 2. Summary of the observational characteristics predicted by the standard solar HENA model at Earth.

\begin{tabular}{|c|c|c|c|}
\hline \multicolumn{2}{|c|}{ Species } & Hydrogen & Comments \\
\hline \multicolumn{2}{|c|}{ Energy range } & $5 \mathrm{keV}-20 \mathrm{MeV}$ & Discrete, simulated energies \\
\hline \multicolumn{2}{|c|}{ Vantage point } & Earth & $90^{\circ}$ to shock axis \\
\hline \multicolumn{2}{|c|}{ Angular spread } & $<0.018$ steradian $(\mathrm{sr})$ & $\begin{array}{c}\text { Angular spread of HENAs seen at the Earth } \\
\text { during the shock expansion }\end{array}$ \\
\hline \multirow{2}{*}{$\begin{array}{l}\text { Differential flux } \\
\text { range }\end{array}$} & Lowest peak & $\begin{array}{c}1.5 \times 10^{-5} \mathrm{~cm}^{-2} \mathrm{~s}^{-1} \mathrm{keV}^{-1} \text { at } 20 \\
\mathrm{MeV}\end{array}$ & \multirow{2}{*}{$\begin{array}{l}\geq 1 \mathrm{MeV} \text {. Flux integrated over } \\
\text { the direction distribution above }\end{array}$} \\
\hline & Highest peak & $2 \times 10^{-4} \mathrm{~cm}^{-2} \mathrm{~s}^{-1} \mathrm{keV}^{-1}$ at $1 \mathrm{MeV}$ & \\
\hline \multicolumn{2}{|c|}{ Time span } & $\begin{array}{l}\sim 0.7-20 \text { hrs after the shock } \\
\text { initiation }\end{array}$ & $\begin{array}{c}\text { From the peak of the } 20 \mathrm{MeV} \text { HENAs to the shock } \\
\text { arrival } / 20 \mathrm{keV} \text { HENAs }\end{array}$ \\
\hline
\end{tabular}


Table 3. Comparison of summary characteristics of various parameters with the baseline model. The values in other runs that have higher (lower) values than in the baseline model are shown in different shades of green (red), larger differences are shown in darker colors.

\begin{tabular}{|c|c|c|c|c|}
\hline \multicolumn{2}{|c|}{ Cases $\downarrow /$ Results $\rightarrow$} & \multicolumn{2}{|c|}{$\begin{array}{l}\text { HENA differential flux } \\
\text { range between } 1 \mathrm{MeV} \\
\text { and } 20 \mathrm{MeV} \\
\left(\mathrm{cm}^{-2} \mathrm{~s}^{-1} \mathrm{keV}^{-1}\right)\end{array}$} & \multirow{2}{*}{$\begin{array}{c}\text { Maximal } \\
\text { angular } \\
\text { spread seen } \\
\text { at } 1 \mathrm{AU}(\mathrm{sr}) \\
0.018\end{array}$} \\
\hline Base & ine & $1.5 \mathrm{E}-5$ & $2.0 \mathrm{E}-4$ & \\
\hline \multirow{2}{*}{ Width angle } & $120^{\circ}$ & $2.0 \mathrm{E}-4$ & $1.0 \mathrm{E}-3$ & 0.036 \\
\hline & $180^{\circ}$ & $3.0 \mathrm{E}-5$ & $1.5 \mathrm{E}-3$ & 0.054 \\
\hline \multirow{4}{*}{$\begin{array}{l}\text { Propagation } \\
\text { direction }\end{array}$} & $0^{\circ}$ & $2.0 \mathrm{E}-6$ & $1.5 \mathrm{E}-4$ & 0.041 \\
\hline & $45^{\circ}$ & $5.0 \mathrm{E}-6$ & $2.0 \mathrm{E}-4$ & 0.025 \\
\hline & $135^{\circ}$ & $3.0 E-6$ & $2.5 E-5$ & 0.019 \\
\hline & $180^{\circ}$ & $3.0 \mathrm{E}-7$ & $3.0 \mathrm{E}-6$ & 0.021 \\
\hline \multirow{2}{*}{$\begin{array}{l}\text { Shock } \\
\text { speed }\end{array}$} & $2000 \mathrm{~km} / \mathrm{s}$ & $1.5 \mathrm{E}-5$ & $2.0 \mathrm{E}-4$ & \multirow{2}{*}{0.018} \\
\hline & $2500 \mathrm{~km} / \mathrm{s}$ & $1.5 \mathrm{E}-5$ & $2.0 \mathrm{E}-4$ & \\
\hline \multirow{3}{*}{$\begin{array}{l}\text { Coronal } \\
\text { density }\end{array}$} & $\times 10$ & $4.0 \mathrm{E}-4$ & $1.0 \mathrm{E}-3$ & \multirow{3}{*}{0.018} \\
\hline & $\times 3$ & $8.0 \mathrm{E}-5$ & $4.0 \mathrm{E}-4$ & \\
\hline & 13 & $1.7 \mathrm{E}-6$ & $5.0 \mathrm{E}-5$ & \\
\hline \multirow{3}{*}{ Abundances } & $\begin{array}{l}\mathrm{nO}^{6+} / \mathrm{np} \\
=2 \times 10^{-3}\end{array}$ & $3.0 \mathrm{E}-5$ & $4.0 \mathrm{E}-4$ & \multirow{3}{*}{0.018} \\
\hline & $\begin{array}{l}\mathrm{nO}^{6+} / \mathrm{np} \\
=2 \times 10^{-4}\end{array}$ & $3.0 \mathrm{E}-6$ & $4.0 E-5$ & \\
\hline & $\begin{array}{c}\mathrm{nC}^{4+} / \mathrm{nO}^{6+} \\
=0.2\end{array}$ & $1.5 \mathrm{E}-5$ & $2.0 \mathrm{E}-4$ & \\
\hline
\end{tabular}

baseline model. These differences are due to the enhanced production and the Earthward-propagating part of the downstream shocked solar wind.

An even wider width angle, e.g., $180^{\circ}$, does not cause an increase in the detected HENA flux that is proportional to the angular spread of the source region, especially for the more energetic HENAs. We will discuss the reason in the next section.

\subsection{Earthward Shock Direction}

Another typical situation is when CMEs propagate towards the

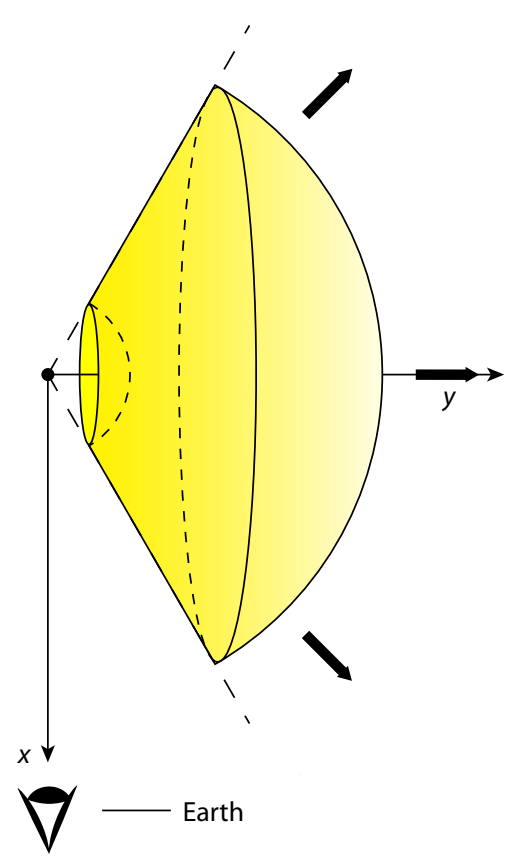

Figure 5. Illustration of the shock with a $120^{\circ}$ cone width angle.

Earth, which is in a propagation direction of $0^{\circ}$ (Figure 7, Table 5). The simulation results are shown in Figure 8. The SEP HENA flux in the Earthward case is lower than that in the baseline model. The SEP HENAs also arrive 10 minutes later than in the baseline model. Both changes are due to increased HENA losses in the lower corona while the HENA production remains the same. Compared to the baseline model, HENAs in the Earthward case travel much longer in the downstream region of the shock, where the compressed solar wind causes a higher proton impact ionization rate, the dominant loss process in the corona. Therefore, the HENA survival probability is significantly lower in the Earthward case than in the baseline model. The result is thus a reduction of SEP HENA flux in the Earthward case. In other words, the lower corona is optically thick for SEP HENA propagation, and the optical thickness for SEP HENAs is greater in the Earthward case than that in the baseline model due to different geometries. The lower corona is optically thicker for the more energetic HENAs, so the earliest high-energy HENAs rarely escape the source region. This also ex-
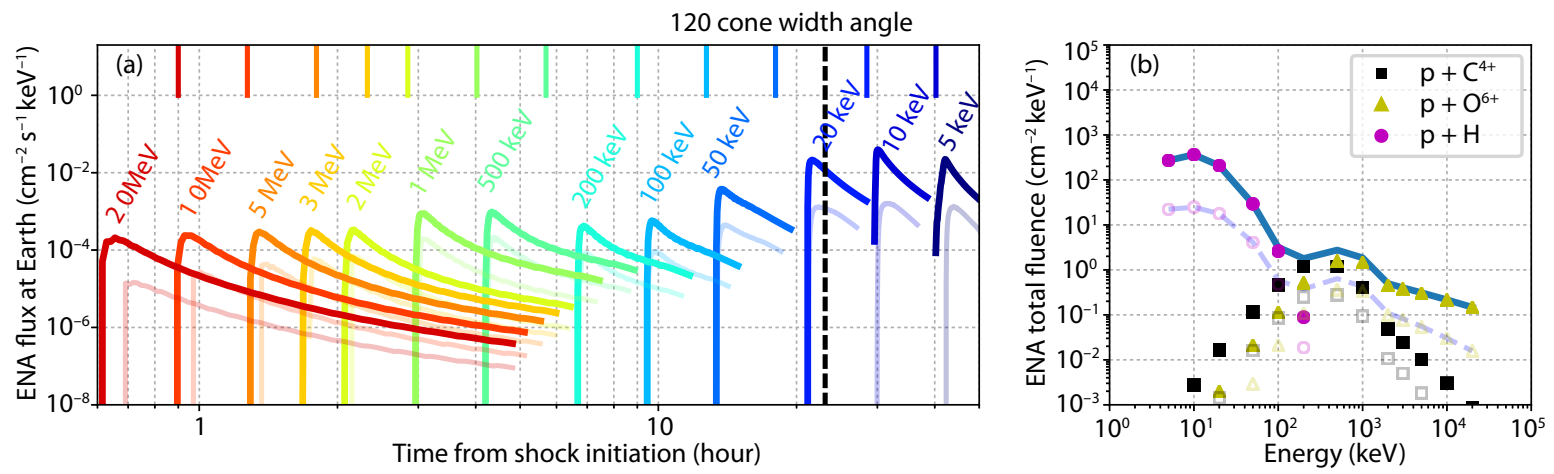

Figure 6. HENA characteristics at the Earth in the case of a $120^{\circ}$ shock width angle. Semi-transparent lines are from the baseline model, for comparison. The format is the same as Figure 4. 
Table 4. Comparison of results from the $120^{\circ}$ and $180^{\circ}$ width angle cases.

\begin{tabular}{lcc}
\hline \multicolumn{1}{c}{ Case } & $120^{\circ}$ width angle & $180^{\circ}$ width angle \\
\hline $\begin{array}{l}\text { Differential flux range } \\
\text { before SEP arrival }\end{array}$ & $\begin{array}{c}2 \times 10^{-4}-10^{-3} \\
\mathrm{~cm}^{-2} \mathrm{~s}^{-1} \mathrm{keV}^{-1}\end{array}$ & $\begin{array}{c}3 \times 10^{-5}-1.5 \times 10^{-3} \\
\mathrm{~cm}^{-2} \mathrm{~s}^{-1} \mathrm{keV}^{-1}\end{array}$ \\
\hline Angular spread & $\begin{array}{l}\text { Source region seen } \\
\text { at } 1 \mathrm{AU},<0.054 \mathrm{sr}\end{array}$ & $\begin{array}{l}\text { Source region seen } \\
\text { at } 1 \mathrm{AU},<0.036 \mathrm{sr}\end{array}$ \\
\hline Time span & $\sim 0.7-20 \mathrm{hrs}$ after the shock onset \\
\hline
\end{tabular}

plains why the $180^{\circ}$ width angle case has lower SEP HENA fluxes than in the $120^{\circ}$ width angle case: the difference is due to the Earthward-propagating part of the shock.

An even lower HENA flux than in the baseline model would suggest that use of HENAs resulting from Earthward-propagating CMEs to forecast SEPs might be difficult. However, favorable conditions such as a wider shock width angle or higher local density could nevertheless make such forecasting feasible.

\subsection{Higher Coronal Density}

The coronal density is highly variable. This variability includes the large-scale spatial structure, from streamers to coronal holes, and temporal variation ranging from solar flares to the activity cycle. The extent of variability easily reaches 1-2 orders of magnitude (e.g., Aschwanden, 2005, p. 25). The baseline model uses a moder-

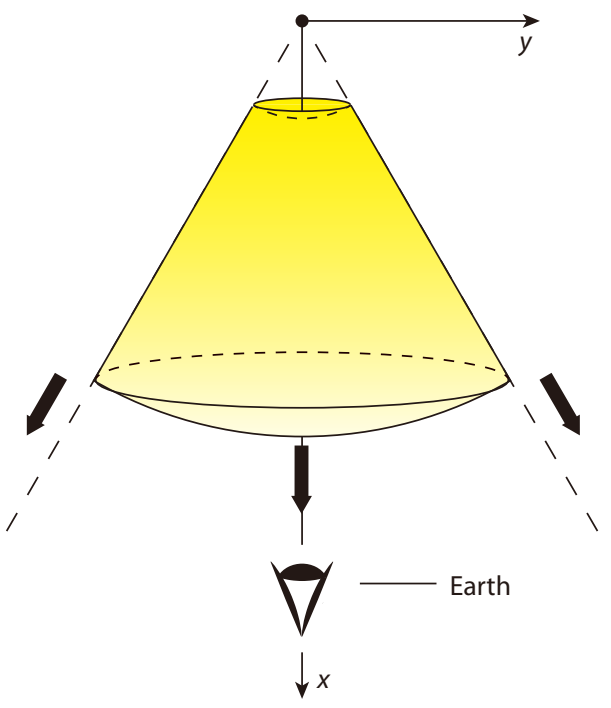

Figure 7. Illustration of the case of Earthward propagation direction.

ate value for the background coronal density; in this study, we tested cases with densities ranging from 0.1 to 10 times the baseline model value. The geometric configuration, in this case, is the same as the baseline model and is not shown here again.

Figure 9 presents results for the simulation case of 10 times coronal density (Table 6). The most noteworthy difference from the
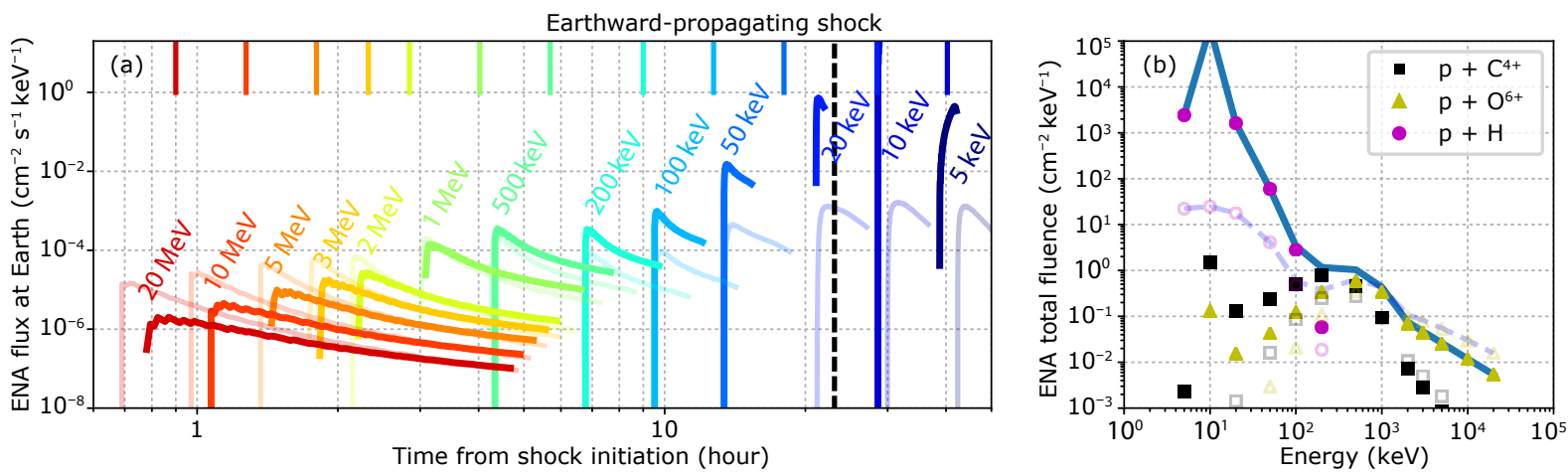

Figure 8. HENA characteristics at the Earth in the case of Earthward shock propagation. Semi-transparent lines and symbols are from the baseline model for easy comparison. The format is the same as Figure 4. The black dashed vertical line shows the arrival time of the shock at the Earth.
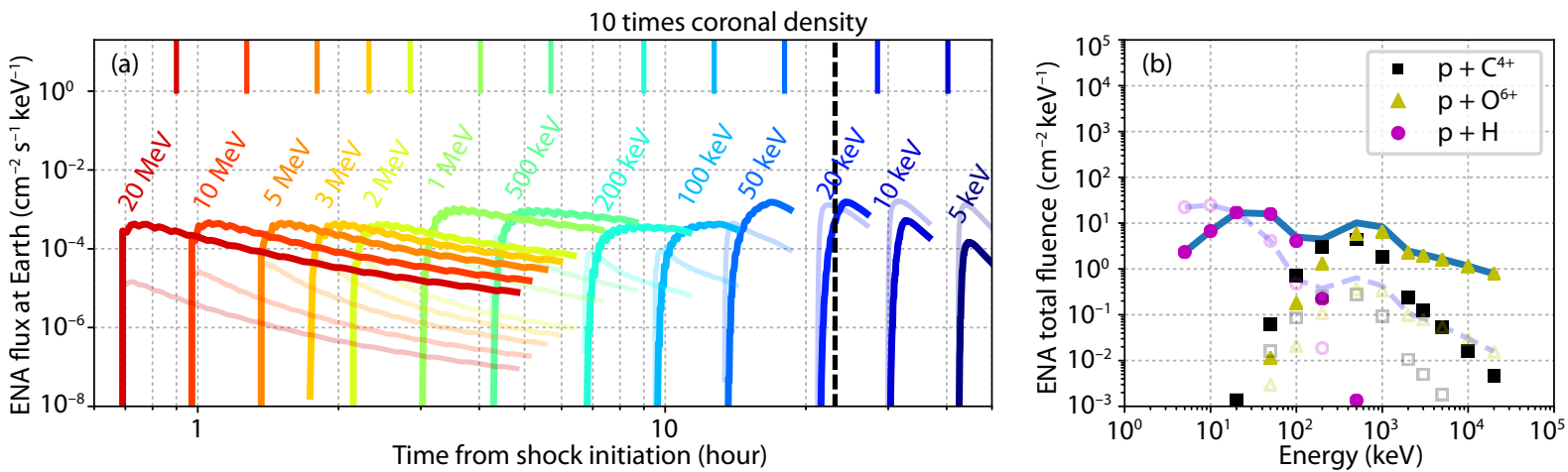

Figure 9. HENA characteristics at the Earth in the case of 10 times the coronal density. Semi-transparent lines and symbols are from the baseline model for easy comparison. The format is the same as Figure 4. 
Table 5. Results from the $0^{\circ}$ propagation direction cases.

\begin{tabular}{lc}
\hline \multicolumn{1}{c}{ Case } & $0^{\circ}$ propagation direction \\
\hline $\begin{array}{l}\text { Differential flux range } 1-20 \mathrm{MeV} \\
\text { before SEP arrival }\end{array}$ & $\begin{array}{c}2 \times 10^{-6}-3 \times 10^{-5} \\
\mathrm{~cm}^{-2} \mathrm{~s}^{-1} \mathrm{keV}^{-1}\end{array}$ \\
\hline Angular spread & $\begin{array}{c}\text { Source region seen at } \\
1 \mathrm{AU},<0.041 \mathrm{sr}\end{array}$ \\
\hline Time span & $\sim 0.8-20$ hrs after \\
& shock onset \\
\hline
\end{tabular}

Table 6. Results from comparing the $\times 10$ and $\times 3$ coronal density cases.

\begin{tabular}{lcc}
\hline \multicolumn{1}{c}{ Case } & $\begin{array}{c}10 \text { times coronal } \\
\text { density }\end{array}$ & $\begin{array}{c}3 \text { times coronal } \\
\text { density }\end{array}$ \\
\hline $\begin{array}{l}\text { Differential flux range } \\
\text { 1-20 MeV before } \\
\text { SEP arrival }\end{array}$ & $\begin{array}{c}5 \times 10^{-4}-7 \times 10^{-4} \\
\mathrm{~cm}^{-2} \mathrm{~s}^{-1} \mathrm{keV}^{-1}\end{array}$ & $\begin{array}{c}1.8 \times 10^{-4}-3 \times 10^{-4} \\
\mathrm{~cm}^{-2} \mathrm{~s}^{-1} \mathrm{keV}^{-1}\end{array}$ \\
\hline Angular spread & $\begin{array}{c}\text { Source region seen at } \\
1 \mathrm{AU},<0.018 \mathrm{sr}\end{array}$ \\
\hline Time span & $\sim 0.7-20 \mathrm{hrs}$ after shock onset \\
\hline
\end{tabular}

baseline model is the drastic increase in SEP HENAs: almost 10 times the differential flux of the baseline model. This average ratio is energy dependent: at $20 \mathrm{MeV}$ the ratio is more than 20 , while at $1 \mathrm{MeV}$ it is close to 5 . The increase in HENA flux is caused by the combined effects of the increased production and loss rates, since the geometric configuration of the high coronal density case is identical to the baseline model. The production rate is proportional to the square of the coronal density due to the fact that all production processes contain two reactants that are proportional to the total coronal density (Figure 2). The loss rate, on the other hand, is a combination of the density-independent process (photoionization) and density-dependent processes (charge exchange and impact ionization). The density-dependent processes dominate in the lower corona region because the coronal density decays faster with distance than the reversed square law (Equation (2)). As a result, in the lower corona HENAs of lower energies suffer greater losses than do higher energy HENAs, causing the energy-dependent flux ratios. Nevertheless, a higher HENA flux is easier to observe and thus, when it occurs, may be useful for SEP forecasting purposes.

\section{Discussion of the Feasibility of HENA Measurements Using Current Technology}

We have adopted a simplified production model ("the baseline model") for gradual SEP events. The baseline model was introduced to explain the precursor HENAs observed by STEREO-A and -B spacecraft (Mewaldt et al., 2009; Wang LH et al., 2014). We expanded the parameter space to study the correlation between properties of SEP production processes and the properties of the resulting HENAs observed at the Earth. The purpose was to assess the applicability of solar HENA data to forecasting the arrival of solar SEP events at Earth orbit. Our results show that some parameter values can cause higher solar HENA fluxes than those in the baseline model, values sufficiently high that SEP forecasting might be feasible.

\subsection{Solar HENA and SEP Forecast}

Here we propose that the monitoring of solar HENAs with energies exceeding $1 \mathrm{MeV}$ can potentially be a new proxy in the forecasting of SEP events. The established and currently developing approaches for short lead-time forecasts of SEP events rely mainly on the observation of relativistic electrons and photons from hard $\mathrm{X}$-ray to radio frequencies to monitor solar eruptive events. These approaches rely on statistical correlations or physical models (cf., Klein and Dalla, 2017) to correlate the particle and photon observations with SEP properties. We propose that solar HENAs at MeV energies, which are closely associated with SEPs in the solar corona, have a similar potential as a new proxy for SEP forecasting, or at least could provide additional information to improve SEP forecasting by other models.

First, solar HENAs arrive earlier than the associated SEPs due to their straight-line propagation compared to the curved magnetic field path followed by charged energetic particles (Reames, 1997). In our simulation results, solar HENAs may take $\sim 20 \%$ shorter time to reach the Earth than their parent SEPs. This means 10 minutes lead time for $20 \mathrm{MeV}$ particles. The exact lead time depends on the energy range, the shock source location, and its magnetic connectivity to the Earth. Therefore, by monitoring solar HENAs in concerned SEP energy range, we may be able to predict whether a solar eruptive event is likely to produce SEPs. Secondly, solar HENAs provide new information to constrain some plasma properties at the SEP source region inverted by other models. The energy spectrum of HENAs remains the same as they travel in space; therefore the observed spectrum is very similar to the energy spectrum at the HENA source region, which is also the SEP source region. If other established or prospective models predict the SEP energy spectrum and solar corona properties at the source region, one can then deduce the solar HENA spectrum and compare it with the observed spectrum. Such comparisons will help constrain the outputs of these approaches, as mentioned above, to improve the accuracy and level of detail of SEP event forecasts.

\subsection{Observation Feasibility Using Mature Technology}

We assess the feasibility of monitoring SEP HENAs by comparing the predicted SEP HENA flux with the performance of flightproven ENA instruments as a reference. A flight-proven instrument that best matches this energy range is the lon and Neutral CAmera (INCA) on the Cassini spacecraft. INCA measures ENAs between $7 \mathrm{keV}$ and $3 \mathrm{MeV}$ per nucleus with the technology of foilbased time-of-flight measurement with coincidence registration (Mitchell et al., 1993). INCA has a relatively large geometric factor $\left(\sim 0.007 \mathrm{~cm}^{2} \mathrm{sr}\right.$ for an $8^{\circ} \times 4^{\circ}$ pixel) to register the ENAs emitted from the Saturnian system. Table 7 summarizes the key requirements of SEP HENA monitoring and the corresponding performance parameters of INCA as the reference instrument.

The comparison above suggests that there are no critical technology gaps that would prevent the use of INCA's capabilities in monitoring solar HENA for forecasting purposes, despite the fact that INCA was designed more than 25 years ago. With modern technologies such as particle detectors with lower noise levels, faster and more robust electronics, and progress in micro-manufacturing and machining, we conclude that it is feasible to fore- 
Table 7. Comparison between measurement requirements of solar HENAs for SEP forecasting with Cassini/INCA specifications.

\begin{tabular}{|c|c|c|c|}
\hline Solar HENA & $\begin{array}{l}\text { Requirements } \\
\text { for SEP } \\
\text { forecasting }\end{array}$ & $\begin{array}{c}\text { INCA } \\
\text { performance }\end{array}$ & Comments \\
\hline Energy range & $1-20 \mathrm{MeV}$ & $7 \mathrm{keV}-3 \mathrm{MeV}$ & $\begin{array}{l}\text { Req. met with } \\
\text { slight } \\
\text { modification }\end{array}$ \\
\hline $\begin{array}{l}\text { Energy } \\
\text { resolution }\end{array}$ & $\Delta E / E \sim 0.5$ & $\begin{array}{c}50 \mathrm{~km} / \mathrm{s}(\triangle E / E \\
\sim 4.3 \% \text { at } 7 \mathrm{keV} \\
\sim 0.22 \% \text { at } \\
3 \mathrm{MeV})\end{array}$ & Req. met \\
\hline
\end{tabular}

\begin{tabular}{lll}
\hline Angular & & $8^{\circ} \times 4^{\circ}>50 \mathrm{keV}$ \\
resolution & $\sim<4^{\circ} \times 4^{\circ} \mathrm{a}$ & $8^{\circ} \times 8^{\circ} \sim 20 \mathrm{keV}$ \\
(FWHM) & &
\end{tabular}

\begin{tabular}{llll}
$\begin{array}{l}\text { Instrument } \\
\text { instantaneous }\end{array}$ & $\geq 20^{\circ} \times 20^{\circ \mathrm{a}}$ & $120^{\circ} \times 90^{\circ}$ & $\begin{array}{c}\text { moderate } \\
\text { modification }\end{array}$ \\
\hline FOV (FWHM) & $>1.0 \mathrm{~cm}^{2 \mathrm{~b}}$ & $1.38 \mathrm{~cm}^{2}$ & $\begin{array}{c}\text { Req. in principle } \\
\text { met, subject to } \\
\text { FOV change }\end{array}$
\end{tabular}

Justification: a: FOV defined by the requirement of covering twice the angular distance of $40 R_{S}$ seen from the Earth, and the FOV is divided into $5 \times 5$ pixels. b: The effective area $A_{\text {eff }}$ is defined as $A_{\text {eff }}>$ $C_{\text {req }} /\left(F \cdot t_{\text {acc }}\right) \cdot N_{\text {px }}$, where $C_{\text {req }}=10$ is the required total number of counts in $t_{\text {acc, }} F=0.2 \mathrm{~cm}^{-2} \mathrm{~s}^{-1}$ at $5 \mathrm{MeV}$ is the minimum flux to measure, and $t_{\text {acc }}$ is the accumulation time per pixel, which must be shorter than the time of HENA flux, staying higher than $50 \%$ of its peak for all energies; the shortest time among all energies is $\sim 20$ minutes for $20 \mathrm{MeV}$ HENA. $N_{\mathrm{px}}=25$ is the total number of pixels in the FOV. These values give $A_{\text {eff }}>1.0 \mathrm{~cm}^{2}$. INCA, by comparison, has an effective area of $1.38 \mathrm{~cm}^{2}$. In this calculation, we assume all HENAs are evenly distributed within the $20^{\circ} \times 20^{\circ} \mathrm{FOV}$, while in practice the central pixels would receive higher fluxes and hence more counts.

cast SEP events by using a dedicated HENA instrument to monitor solar HENAs. Such a solar HENA instrument will require energy range and performance characteristics similar to those of INCA, and must be able to tolerate the sun disk in its FOV.

The limitation of the current study is our simplified analytical model for solar HENA production. To advance this work, more realistic numerical models must be employed, using MHD or hybrid simulation of solar HENA distributions. The properties of SEPs at Earth were not explicitly modelled in this work. Combining decades of SEP observations at the Earth and more sophisticated numerical models should make it possible to invert the velocity distributions of the SEPs at birth, and to compare directly the properties of the resultant HENAs to those of the expected SEPs. Such comparisons would be more practical for actual forecasting than using the properties of the shocks.

\section{Conclusions}

STEREO/LET observed a precursor of a large SEP event impacting the Earth. The precursor was interpreted as energetic neutral hydrogen atoms, originating from energetic protons that were neutralized through recombination and charge exchange reactions in the lower corona (Mewaldt et al., 2009; Wang LH et al., 2014). This explanation was later supported with an analytical model describ- ing the production and propagation of these solar HENAs (Wang LH et al., 2014). If the interpretation is correct, the close relation between SEPs and the HENAs that accompany them suggests that monitoring solar HENAs is a promising method for forecasting the arrival of their parent SEPs at Earth orbit.

By expanding the results in Wang LH et al. (2014) to a wider parameter space, we have performed a more comprehensive parameter study modelling solar HENAs. We have also assessed the dependence of solar HENA properties on those of the SEP-generating shocks. We reconstructed the model in Wang LH et al. (2014) and varied parameters such as shock geometry, shock speed, coronal density, and coronal abundances for relevant ion species in the lower corona. The variations cover most of the ranges of their observed values and we did not vary more than one parameter at the same time.

We analyzed the temporal and spectral characteristics of the HENAs arriving at the Earth. The results show that a wider width angle of the shock and higher coronal density will increase the HENA flux before the SEP arrival, because both conditions increase the integral production along the line of sight through the HENA source region. The shock propagating direction has the mixed effect of slightly reducing the HENAs before SEP arrival but increasing the HENAs before the shock arrival. The reason is that, although this propagation direction increases the LOS production rate, the loss rate due to recombination and charge exchange also increases because of the extended propagation distance in the shocked corona.

We propose that solar HENAs could serve as a new proxy in SEP forecasting because of their earlier arrival at the Earth compared to SEPs of the same origin. The typical lead time for $1.8-3.6 \mathrm{MeV}$ SEPs is 2-3 hours. According to the modelled temporal and spectral characteristics of solar HENAs, we have formulated the measurement requirements for solar HENA monitoring. We further compare these measurement requirements with the specifications and performance of a flight-proven instrument with similar energy range: the INCA instrument on the Cassini spacecraft. From this comparison, we conclude that instrument technology is mature enough to build an ENA instrument that satisfies the requirement of monitoring solar HENAs in the Earth orbit.

\section{Acknowledgments}

A part of this work was conducted under the contract 4000113060/14/NL/MV “Energetic Neutrals for Space Environment Monitoring" with ESA. Some of the contents of this paper were taken from the final report of this ESA contract.

\section{References}

Allen, L. A., Habbal, S. R., and Li, X. (2000). Thermal coupling of protons and neutral hydrogen with anisotropic temperatures in the fast solar wind. J. Geophys. Res. :Space Phys., 105(A10), 23123-23134. https://doi.org/10.1029/ 1999JA000437

Andersen, L. H., and Bolko, J. (1990). Radiative recombination between fully stripped ions and free electrons. Phys. Rev. A, 42(3), 1184-1191. https://doi.org/10.1103/PhysRevA.42.1184

Aschwanden, M. J. (2005). Physics of the Solar Corona: an Introduction with Problems and Solutions. Chichester, UK: Praxis Publishing Ltd. 
https://doi.org/10.1007/3-540-30766-4

Barnett, C. F. , Hunter, H. T. , Kirkpatrick, M. I. , Alvarez, I. , Cisneros, C. , and Phaneuf, R. A. (1990). Atomic data for fusion volume 1: collisions of $H, H 2$, $\mathrm{He}$ and $\mathrm{Li}$ atoms and ions with atoms and molecules. ORNL-6086/V1, Oak Ridge, Tennessee: Oak Ridge National Laboratory.

D'Amicis, R., Orsini, S., Antonucci, E., Di Lellis, A. M., Hilchenbach, M., Telloni, D., Mura, A., Milillo, A., Fineschi, S., and Bruno, R. (2007). Numerical simulations of coronal hole-associated neutral solar wind as expected at the Solar Orbiter position. J. Geophys. Res. :Space Phys., 112(A6), A06110. https://doi.org/10.1029/2006JA011969

Gruntman, M. (1997). Energetic neutral atom imaging of space plasmas. Rev. Sci. Instrum., 68(10), 3617-3656. https://doi.org/10.1063/1.1148389

Gruntman, M., Roelof, E. C., Mitchell, D. G., Fahr, H. J., Funsten, H. O., and McComas, D. J. (2001). Energetic neutral atom imaging of the heliospheric boundary region. J. Geophys. Res. :Space Phys., 106(A8), 15767-15781. https://doi.org/10.1029/2000JA000328

Gruntman, M. A. (1994). Neutral solar wind properties: advance warning of major geomagnetic storms. J. Geophys. Res. :Space Phys., 99(A10), 19213-19227. https://doi.org/10.1029/94JA01571

Hsieh, K. C. , Shih, K. L. , McComas, D. J. , Wu, S. T. , and Wu, C. (1992). Forecasting the arrival of fast coronal-mass ejecta at Earth by the detection of 2-20keV neutral atoms. In Proceedings of SPIE 1744, Instrumentation for Magnetospheric Imagery (pp. 72-78). San Diego: SPIE. https://doi.org/10.1117/12.60581

Kahler, S. W. (2001). The correlation between solar energetic particle peak intensities and speeds of coronal mass ejections: effects of ambient particle intensities and energy spectra. J. Geophys. Res. :Space Phys., 106(A10), 20947-20955. https://doi.org/10.1029/2000JA002231

Klein, K. L., and Dalla, S. (2017). Acceleration and propagation of solar energetic particles. Space Sci. Rev., 212(3-4), 1107-1136. https://doi.org/10.1007/s11214-017-0382-4

Kuang, Y. R. (1992). Electron capture by protons and alpha particles from twoelectron targets. J. Phys. B:At. Mol. Opt. Phys., 25(1), 199-211. https://doi.org/10.1088/0953-4075/25/1/023

Lamy, P. L., Floyd, O., Boclet, B., Wojak, J., Gilardy, H., and Barlyaeva, T. (2019). Coronal mass ejections over solar cycles 23 and 24. Space Sci. Rev. , 215(5), 39. https://doi.org/10.1007/s11214-019-0605-y

Lee, M. A. (2005). Coupled hydromagnetic wave excitation and ion acceleration at an evolving coronal/interplanetary shock. Astrophys. J. Suppl. S., 158(1), 38-67. https://doi.org/10.1086/428753

Mewaldt, R. A., Leske, R. A., Stone, E. C., Barghouty, A. F., Labrador, A. W., Cohen, C. M. S., Cummings, A. C., Davis, A. J., von Rosenvinge, T. T., and Wiedenbeck, M. E. (2009). STEREO observations of energetic neutral hydrogen atoms during the 2006 December 5 solar flare. Astrophys. J., 693(1), L11-L15. https://doi.org/10.1088/0004-637X/693/1/L11

Mitchell, D. G., Cheng, A. F., Krimigis, S. M., Keath, E. P., Jaskulek, S. E., Mauk, B. H., McEntire, R. W., Roelof, E. C., Williams, D. J., ... Drake, V. A. (1993). INCA: the ion neutral camera for energetic neutral atom imaging of the Saturnian magnetosphere. Opt. Eng., 32(12), 3096-3101. https://doi.org/10.1117/12. 155609

Reames, D. V. (1997). Energetic particles and the structure of coronal mass ejections. In N. Crooker, et al. (Eds. ), Coronal Mass Ejections, Volume 99 (pp. 217-226). Washington, DC: American Geophysical Union. https://doi.org/10.1029/GM099p0217

von Steiger, R., Schwadron, N. A., Fisk, L. A., Geiss, J., Gloeckler, G., Hefti, S., Wilken, B., Wimmer-Schweingruber, R. R., and Zurbuchen, T. H. (2000). Composition of quasi-stationary solar wind flows from Ulysses/Solar Wind Ion Composition Spectrometer. J. Geophys. Res. :Space Phys., 105(A12), 27217-27238. https://doi.org/10.1029/1999JA000358

Wang, L. H., Li, G., Shih, A. Y., Lin, R. P., and Wimmer-Schweingruber, R. F. (2014). Simulation of energetic neutral atoms from solar energetic particles. Astrophys. J. Lett., 793(2), L37. https://doi.org/10.1088/2041-8205/793/2/L37 Wurz, P. , and Gabriel, A. (1999). Working group 4: wind acceleration processes. In Proceedings of the 8th SOHO Workshop: Plasma Dynamics and Diagnostics in the Solar Transition Region and Corona (pp. 87). Paris, France: ESA, NASA, C. N. R. S. -I. N. S. U. 